\title{
Electronic cigarette use is not associated with quitting of conventional cigarettes in youth smokers
}

\author{
Man Ping Wang ${ }^{1}$, William H. Li ${ }^{1}$, Yongda $\mathrm{Wu}^{1}$, Tai Hing $\mathrm{Lam}^{2}$ and Sophia S. Chan ${ }^{1}$
}

BACKGROUND: To investigate the association between electronic cigarette (e-cig) use and smoking cessation among smokers who called the Youth Quitline in Hong Kong.

METHODS: This longitudinal study collected data on youth smokers' ( $N=189)$ use and perception of e-cigs, conventional cigarette smoking behavior, and sociodemographic characteristics at baseline. Self-reported past 7-day point prevalence of abstinence (PPA) was assessed in the 6-month telephone follow-up. Linear and logistic regressions were used to estimate the association of e-cig use with quitting cigarette smoking and other cessation-related outcomes.

RESULTS: E-cig users were younger, more addicted to nicotine, and less ready to quit (all $P<0.05$ ) at baseline. The PPA rate was lower in e-cig users (13.4\% vs. 20.8\%) at followup. E-cig use was not associated with PPA at the 6-month follow-up (odds ratio (OR): 0.56, 95\% Cl: 0.24 to 1.35), but it was nonsignificantly related to more cessation attempts (raw coefficient (b): $1.26,95 \% \mathrm{Cl}$ : -0.13 to 2.66 ). Among those who still smoked, e-cig use was nonsignificantly associated with intention to quit smoking (OR: $0.55,95 \% \mathrm{Cl}$ : 0.15 to 2.05 ), nicotine dependence (Fagerström score, b: 0.75, 95\% Cl: -0.39 to 1.90), and perceptions on quitting cigarette smoking.

CONCLUSION: E-cig use was not associated with successful smoking cessation among Youth Quitline smokers.

E lectronic cigarettes (e-cigs) are popular worldwide, especially among the youth. A population survey in 2012 showed that Europeans aged 15-24 years had 230\% higher odds of having ever used e-cigs (1). The prevalence of e-cig use even approached that of conventional cigarettes. A recent study among high-school students in the United States reported that the prevalence of e-cig use in the past 30 days increased from $1.7 \%$ in 2011 to $7.7 \%$ in 2013 , whereas the proportion of current conventional cigarette smokers dropped from 15.1 to $13.1 \%$ (ref. 2). E-cig use in Asian countries is emerging with 9.4\% and $4.7 \%$ of Korean adolescents having ever tried and used it in the past 30 days, respectively (3). In Hong Kong, the prevalence of past 30-day e-cig use in adolescents is relatively low (1.1\% in 2012-2013), but it is gradually approaching that of conventional cigarette use (3.3\%) (4).
E-cig use is more prevalent among current adolescent smokers $(1,3,4)$. Smoking cessation is one of the main reasons reported for the use of e-cigs (5). The effects of e-cigs on smoking cessation among adolescents are uncertain because only two cross-sectional studies have reported results on successful abstinence $(3,6)$. Both ever and past 30-day e-cig use were associated with lower abstinence rate at 30-day, 6-month, and 1-year follow-ups among ex-smokers from a representative sample of US secondary students (6). Similar results were found in Korean adolescents using e-cigs in the past 30 days (3). Prospective studies are needed to better assess the effects of e-cigs on quitting cigarette smoking among adolescents. Adult studies found inconclusive evidence on the effectiveness of e-cigs as a smoking cessation aid (7). A recent meta-analysis concluded that e-cig users had $28 \%$ lower odds of quitting (8), but the designs of the studies included varied, which rendered this result less convincing. Meanwhile, positive associations were also reported by a paper that included three cross-sectional studies among US young adult smokers (aged 18-25 years), in which one study found that past-month e-cig use was positively related to pastyear quitting attempts and another found a positive association with the desire to quit (9).

E-cig may affect the cessation of cigarette smoking differently between adolescents and adults as similar phenomena were observed with reference to the use of medication for smoking cessation (e.g., nicotine replacement therapy and varenicline). A recent systematic review failed to find long-term beneficial effects of smoking medication use among adolescents (10). The e-cig was widely promoted as a smoking cessation tool worldwide and in Hong Kong, and it is available on the internet and in some local shops. The nicotine-containing e-cig is required to be registered according to the Pharmacy and Poisons Ordinance, but none has done so as of December 2016. In addition, the use of e-cigs, irrespective of whether it contains nicotine, is prohibited in smoke-free areas under the comprehensive smoke-free legislation. We investigated the prospective associations of e-cig use with smoking cessation behaviors, level of nicotine dependence, and perceived selfefficacy on quitting cigarette smoking in youth smokers in Hong Kong.

\footnotetext{
${ }^{1}$ School of Nursing, University of Hong Kong, Hong Kong; ${ }^{2}$ School of Public Health, University of Hong Kong, Hong Kong. Correspondence: Man Ping Wang (mpwang@hku.hk) Received 23 November 2016; accepted 11 March 2017; advance online publication 24 May 2017. doi:10.1038/pr.2017.80
} 
Table 1. Baseline sociodemographic characteristics and smoking behavior by ever e-cig use in all participants $(N=189)$

\begin{tabular}{|c|c|c|c|c|}
\hline & \multirow[t]{2}{*}{ All (\%) } & \multicolumn{3}{|c|}{ E-cig use } \\
\hline & & Nonuser (\%) & User (\%) & $P$ value \\
\hline Male & 82.0 & 79.2 & 83.9 & 0.41 \\
\hline Age (mean $\pm S D)$, years & $18.1 \pm 2.7$ & $18.9 \pm 2.7$ & $17.5 \pm 2.6$ & $<0.001$ \\
\hline Highest education & & & & $<0.01$ \\
\hline Junior second or below & 24.3 & 12.5 & 32.7 & \\
\hline Senior second or diploma & 57.2 & 59.7 & 55.5 & \\
\hline Sub-degree/higher diploma or above & 18.5 & 27.8 & 11.9 & \\
\hline Work/study status & & & & 0.49 \\
\hline Full-time student & 74.6 & 69.3 & 78.3 & \\
\hline Employed or self-employed & 16.6 & 18.7 & 15.1 & \\
\hline Unemployed & 1.7 & 2.7 & 0.9 & \\
\hline Others & 7.2 & 9.3 & 5.7 & \\
\hline Number of cigarettes consumed per day ${ }^{\mathrm{a}}$ (mean $\pm \mathrm{SD}$ ) & $11.5 \pm 9.6$ & $9.4 \pm 8.1$ & $12.9 \pm 10.3$ & 0.02 \\
\hline Nicotine dependence $^{b}($ mean $\pm S D)$ & $2.8 \pm 2.4$ & $2.2 \pm 2.2$ & $3.3 \pm 2.4$ & $<0.01$ \\
\hline Readiness to quit smoking & & & & 0.03 \\
\hline Action/preparation & 51.4 & 60.8 & 44.8 & \\
\hline Contemplation/pre-contemplation & 48.6 & 39.2 & 55.2 & \\
\hline Intention to quit & 94.7 & 96.1 & 93.8 & 0.48 \\
\hline Lifetime quit attempts (mean $\pm S D$ ) & $3.3 \pm 5.3$ & $3.2 \pm 5.3$ & $3.3 \pm 5.3$ & 0.91 \\
\hline \multicolumn{5}{|l|}{ Perceived self-efficacy in quitting smoking ${ }^{c}$ (mean $\left.\pm S D\right)$} \\
\hline Importance in successfully quitting cigarette smoking & $7.3 \pm 2.2$ & $7.6 \pm 2.2$ & $7.2 \pm 2.3$ & 0.25 \\
\hline Confidence in successfully quitting cigarette smoking & $6.1 \pm 2.3$ & $6.2 \pm 2.4$ & $6.0 \pm 2.2$ & 0.56 \\
\hline Difficulties in successfully quitting cigarette smoking & $3.6 \pm 2.3$ & $3.6 \pm 2.6$ & $3.5 \pm 2.1$ & 0.72 \\
\hline
\end{tabular}

\section{RESULTS}

The participants included in the study shared similar sociodemographic characteristics with excluded smokers, except that they were younger ( 18.1 vs. 19.6 years, $P<0.01$; data not shown in the tables). Most participants (82.0\%) were male, with a mean age of $18.1( \pm 2.7)$ years, and $75.7 \%$ had an education level of high school or above (Table 1). The average number of cigarettes consumed per day on weekends was 11.5 $( \pm 9.6)$ and level of nicotine dependence was mild (Fagerström score: $2.8 \pm 2.4$ ). As expected, nearly all smokers $(94.7 \%)$ had an intention to quit and over half of the smokers (51.4\%) were more ready to abstain from smoking. Smokers generally perceived successful quitting as important $(7.3 \pm 2.2)$, not difficult $(3.6 \pm 2.3)$, and they were confident in their ability to quit $(6.1 \pm 2.3)$. A total of 112 smokers $(59.3 \%, 95 \%$ CI: 51.9 to $66.3 \%$ ) had ever tried an e-cig and they were younger (17.5 vs. 18.9 years), more addicted to nicotine (Fagerström score: 3.3 vs. 2.2 ), less ready to quit (44.8 vs. $60.8 \%$ ), and consumed more cigarettes per day on weekends (12.9 vs. 9.4), compared with nonusers (all $P<0.05$ ).
Table 2 shows that 31 (16.4\%, 95\% CI: 11.1 to $21.7 \%)$ participants reported abstinence at the 6-month follow-up with a lower PPA for the past 7 days for e-cig users than that for nonusers (13.4 vs. 20.8\%). E-cig users had nonsignificant lower odds of quitting (adjusted OR: 0.56, 95\% CI: 0.24 to 1.35), although they appeared to have tried quitting more frequently ( $b: 1.26,95 \% \mathrm{CI}:-0.13$ to 2.66$)$. Among those who remained smokers at the 6-month follow-up, ever e-cig use was nonsignificantly associated with lower intention to quit (adjusted OR: 0.55, 95\% CI: 0.15 to 2.05; Table 3), higher level of nicotine dependence ( $b: 0.75,95 \% \mathrm{CI}:-0.39$ to 1.90$)$, more cigarette consumption ( $b: 1.47,95 \% \mathrm{CI}:-2.21$ to 5.15$)$, and lower perceived importance ( $b$ : $-0.76,95 \% \mathrm{CI}:-2.01$ to 0.49$)$, confidence ( $b:-0.13,95 \% \mathrm{CI}:-1.80$ to 1.54$)$, and difficulties (b: $-0.75,95 \%$ CI: -2.38 to 0.88 ) pertaining to quitting cigarette smoking.

\section{DISCUSSION}

To the best of our knowledge, this is the first report on prospective associations of e-cig use on quitting cigarette 
Table 2. Association between ever e-cig use and quitting cigarette smoking and quit attempts in all participants at the 6-month follow-up

\begin{tabular}{|c|c|c|c|c|c|c|}
\hline & \multirow[t]{2}{*}{$N$} & \multicolumn{2}{|c|}{ Outcome $^{a}$} & \multirow[t]{2}{*}{ Effect size ${ }^{b}$} & \multicolumn{2}{|c|}{ Raw coefficient $(95 \% \mathrm{Cl})^{\mathrm{c}}$} \\
\hline & & No E-cig use & E-cig use & & Model 1 & Model $2^{d}$ \\
\hline Quitting cigarette & 189 & $20.8 \%$ & $13.4 \%$ & 0.10 & $0.59(0.27$ to 1.28$)$ & $0.56(0.24 \text { to } 1.35)^{\mathrm{e}}$ \\
\hline Quit attempts & 89 & $2.1 \pm 2.4$ & $3.1 \pm 3.2$ & 0.35 & $1.32(0.05 \text { to } 2.60)^{f}$ & $1.26(-0.13$ to 2.66$)$ \\
\hline
\end{tabular}

$\mathrm{Cl}$, confidence interval.

apercentage for quitting cigarette smoking and mean for quit attempts.

${ }^{b}$ Cohen's d/Cohen's w: 0.10, small; 0.30, medium; and 0.50, large.

'Odds ratio for quitting cigarette smoking and raw coefficients from the linear regression for quit attempts.

dModel 2: adjusting for model 1 variables and sex, age, smoking friends, and smoking family members.

${ }^{\text {e}}$ Adjusting for model 2 variables, and for baseline quit attempts and nicotine dependence level.

${ }^{f}$ Adjusting for lifetime quit attempts reported at baseline.

Table 3. Association between ever e-cig use and quitting-related outcomes in non-quitters at the 6-month follow-up

\begin{tabular}{|c|c|c|c|c|c|c|}
\hline & \multirow[t]{2}{*}{$N$} & \multicolumn{2}{|c|}{ Outcome $(\text { mean } \pm S D)^{a}$} & \multirow[t]{2}{*}{ Effect size ${ }^{b}$} & \multicolumn{2}{|c|}{ Raw coefficient $(95 \% \mathrm{CI})^{\mathrm{c}}$} \\
\hline & & No E-cig use & E-cig use & & Model $1^{\mathrm{d}}$ & Model $2^{\mathrm{e}}$ \\
\hline Intention to quit & 61 & $80.8 \%$ & $68.6 \%$ & 0.14 & 0.51 (0.15 to 1.72$)$ & 0.55 (0.15 to 2.05$)$ \\
\hline Nicotine dependence ${ }^{f}$ & 57 & $1.5 \pm 1.6$ & $2.6 \pm 2.1$ & 0.61 & $1.02(0.00$ to 2.04$)$ & $0.75(-0.39$ to 1.90$)$ \\
\hline Number of cigarettes consumed per day ${ }^{g}$ & 65 & $7.2 \pm 6.4$ & $8.7 \pm 6.9$ & 0.22 & $1.38(-1.98$ to 4.73$)$ & $1.47(-2.21$ to 5.15$)$ \\
\hline \multicolumn{7}{|l|}{ Perceived self-efficacy in quitting smoking ${ }^{\mathrm{h}}$} \\
\hline Importance in successfully quitting cigarette smoking & 56 & $7.9 \pm 1.9$ & $6.6 \pm 2.3$ & 0.59 & $-0.85(-1.93$ to 0.24$)$ & $-0.76(-2.01$ to 0.49$)$ \\
\hline Confidence in successfully quitting cigarette smoking & 55 & $5.0 \pm 2.8$ & $5.3 \pm 2.5$ & 0.09 & $0.06(-1.49$ to 1.62$)$ & $-0.13(-1.80$ to 1.54$)$ \\
\hline Difficulties in successfully quitting cigarette smoking & 50 & $3.8 \pm 2.9$ & $3.2 \pm 2.5$ & 0.25 & $-1.06(-2.69$ to 0.56$)$ & $-0.75(-2.38$ to 0.88$)$ \\
\hline
\end{tabular}

$\mathrm{Cl}$, confidence interval; e-cig, electronic cigarette.

${ }^{a}$ Mean of outcome for all variables except $\%$ for intention to quit.

${ }^{b}$ Cohen's d/Cohen's w: 0.10, small; 0.30, medium; and 0.50, large.

'All are raw coefficients from the linear regression except for intention to quit, which are odds ratios.

${ }^{\mathrm{d}}$ Model 1: adjusting for baseline respective level.

eModel 2: adjusting for model 1 variables and for sex, age, smoking friends, and smoking family members.

fFagerström score: 0-3, mild; 4-5, moderate; and 6-10 severe.

${ }^{9}$ Cigarettes consumed per day on weekends.

hscore: 0-10, higher scores indicate better perceived self-efficacy.

smoking in Youth Quitline smokers. Among these motivated youth smokers, ever e-cig use did not predict abstinence from cigarette smoking. However, the finding of nonsignificant negative associations with nicotine dependence, intention to quit, number of cigarettes consumed per day, and perceived self-efficacy among non-quitters was consistent with that on quitting cigarette smoking. The result on quitting cigarette smoking was consistent with that reported in American and Korean cross-sectional adolescent studies $(3,6)$. Such negative or null associations between e-cig use and intention to quit were also reported by cross-sectional studies among US and Hong Kong adolescents $(2,4,11,12)$. E-cig users appeared to have more quit attempts than did nonusers in our study, which was similar to the positive association between e-cig use and quit attempts in a convenient sample of US young adults (9) and a representative sample of Korean adolescents (3).

The lack of prospective association between e-cig use and smoking cessation may be explained by the fact that only a few smokers (15.5\%) used nicotine-containing e-cigs in our study. Although e-cig use can mimic smoking behaviors, the effect of nicotine-free e-cigs on quitting cigarette is weaker than that of e-cigs containing nicotine in adults (13-15).
New-generation e-cigs, which have improved nicotine delivery systems (16), have been found to be more effective in relieving cravings (17) and promoting cessation (18) in adults. However, nicotine-containing e-cigs may not be effective in adolescents even if such products could indeed help adults quit cigarette smoking, as corroborated by nicotine replacement therapy studies $(10,19)$. However, no study has investigated the cessation effect of nicotinecontaining e-cigs in adolescents. In this regard, a subgroup analysis on nicotine e-cig use is not feasible in the present study because few smokers used such e-cigs.

Another explanation may be that the participants did not engage in e-cig use for aiding smoking cessation because $55.9 \%$ of the smokers in this study planned to quit smoking just by themselves or without a specific method, and only six planned to use e-cigs for quitting cigarettes. Many smokers (67.8\%) did not perceive e-cigs as an effective tool for smoking cessation. Other studies found that non-cessationoriented e-cig users usually stopped using e-cigs after trying them, or used them infrequently $(5,20)$. Cessation-oriented users may be more willing to practice e-cig use for a longer period, which may facilitate learning on how to inhale a 


\section{E-cig use and quitting in youth smokers}

satisfactory level of nicotine (21) and may lead to a higher chance of quitting cigarette smoking.

This study has several limitations. More in-depth analysis was restricted by the lack of detailed use pattern (e.g., number of days of e-cig use in the past 30 days, number of puffs/ session per day, and reason for use). Due to the small sample size, a post-priori analysis showed that the statistical power was 0.27 for the detection of a significant association, if the true effect of ever e-cig use on quitting conventional cigarette was assumed to be OR $=0.59$, as found in this study. Smoking abstinence was self-reported because biochemical validation of quitting cigarette smoking remained low (58.1\% of all quit), mostly due to the participants' busy schedule. As an observational study, unmeasured confounders could not be eliminated, which precluded drawing a causal inference.

Our findings showed that e-cig use was not associated with successful abstinence and it appeared to increase difficulties in quitting cigarette smoking in Youth Quitline smokers. Given the prevalent e-cig use in the youth and other evidence on the adverse effects on smoking initiation and respiratory health $(22,23)$, further public health action should be taken against the promotion, sale, and use of e-cigs.

\section{METHODS}

\section{Design}

We analyzed the longitudinal data from the Youth Quitline, which provides a free smoking cessation service (counseling without pharmacological intervention) for 6 months. This quitline is funded by the Tobacco Control Office, Department of Health, Government of Hong Kong SAR, and is operated by the School of Nursing, University of Hong Kong. Details of this quitline have been reported elsewhere (24). Smokers who initiated a call to the YQL were screened, and those who could speak Cantonese, smoked at least one cigarette in the past 30 days, and were aged $\leq 25$ years were eligible to receive the service. Those unable to communicate or those who were undergoing other smoking cessation programs were excluded. Smokers completed the assessment questionnaire on the phone at baseline, and at the 3-day, 1-week, 2-week, and 1-month follow-ups. Counseling was provided after each assessment, based on the smoker's smoking behaviors, using the "five A's" strategy (ask about smoking status, advise to quit, assess readiness to quit, assist to quit, and arrange follow-up contact) (25). Smoking cessation outcomes were assessed at the 6-month follow-up using self-reported abstinence, which was also validated by a salivary cotinine level of $<10 \mathrm{ng} / \mathrm{ml}$ as an indicator of successful cessation (26). Ethical approval was obtained from the Institutional Review Board of the University of Hong Kong/Hospital Authority West Cluster. Oral informed consent was obtained from all participants.

During 2014-2015, a baseline questionnaire with added questions on e-cig was administered among 224 smokers and 190 replied to the questions on e-cig use (27 had never heard of e-cig and 7 did not answer the questions on e-cig). Follow-ups were successfully conducted with 189 smokers, who were included in the current study. The smokers were asked whether they had ever tried e-cig, even a single puff, and whether they perceived that an e-cig could help them quit smoking. Those who had ever used an e-cig further reported details about its taste (e.g., menthol, fruit, and coffee) and the nicotine concentration of the e-cigs. Sociodemographic characteristics, including age, sex, education, and employment, were recorded. The smoking behaviors assessed included number of smoking friends, number of smokers the youth lived with, number of quit attempts in one's lifetime, whether the smokers wanted to quit (measuring intention to quit, yes/no), planned method, and day determined for quitting cigarette smoking. The Fagerström score, including number of cigarettes consumed per day (weekdays and weekends) and time to first cigarette after waking (27) was calculated. Perceived importance, confidence, and difficulties in successfully quitting cigarette smoking were measured as they predicted abstinence of cigarette use (28). Responses to similar questions on smoking and cessation behaviors were evaluated at the 6-month follow-up, except the response to the question on e-cig use.

\section{Measures}

Smokers who had tried the e-cig at least once were classified as "ever user". Participants rated the perceived effectiveness of an e-cig as a smoking cessation aid on a five-point scale ranging from strongly agree to strongly disagree. Readiness to quit was classified into the following stages with reference to when the respondent planned to quit- action/preparation (already started to quit or planned to quit within 1 month, which indicated a higher readiness to quit) and contemplation/pre-contemplation (planning to quit since longer than 1 month or not yet decided, which indicated a lower readiness to quit) - based on the transtheoretical model of change (29). Level of nicotine dependence was assessed by the Fagerström test and the number of cigarettes consumed per day on weekends because most of the participants were students, who spent most of the weekdays at school, and thus could not smoke ad libitum. Perceived importance, confidence, and difficulties in successfully quitting cigarette smoking were measured on a scale of $0-10$ ((refs 28,30), indicating selfefficacy on quitting (higher scores indicated better perceived selfefficacy). Abstaining from smoking for $>24 \mathrm{~h}$ (ref. 31) (yes/no) was considered a serious quit attempt and the main outcome used was self-reported point prevalence of abstinence (PPA) (32) for the past 7 days at the 6-month follow-up.

\section{Analysis}

Stata (Release 13, StataCorp LP, College Station, TX) was used for the analyses. We adopted a pairwise deletion approach and used a twotailed $\alpha=0.05$ as the significance level. The relationship of sociodemographic characteristics with smoking behaviors and e-cig use was examined. Adjusted ORs for self-reported past 7-day PPA and intention to quit, and raw coefficients $(b)$ from a linear regression for quit attempts, level of nicotine dependence, number of cigarettes consumed per day, and perceived self-efficacy on quitting cigarette smoking in relation to e-cig use were calculated, adjusting for baseline respective levels of smoking behaviors and sociodemographic characteristics when applicable. The effect size was measured by Cohen's $d$ and Cohen's $w$.

\section{ACKNOWLEDGMENTS}

We thank the respondents for participating in the study. The Youth Quitline is funded by Tobacco Control Office, Department of Health, Government of Hong Kong SAR.

Disclosure: The authors declare no conflict of interest.

\section{REFERENCES}

1. Vardavas CI, Filippidis FT, Agaku IT. Determinants and prevalence of e-cigarette use throughout the European Union: a secondary analysis of 26566 youth and adults from 27 Countries. Tob Control 2015;24: $442-8$.

2. Huang L-L, Kowitt SD, Sutfin EL, Patel T, Ranney LM, Goldstein AO. Electronic cigarette use among high school students and its association with cigarette use and smoking cessation, North Carolina Youth Tobacco Surveys, 2011 and 2013. Prev Chronic Dis 2016;13:E103.

3. Lee S, Grana RA, Glantz SA. Electronic cigarette use among Korean adolescents: a cross-sectional study of market penetration, dual use, and relationship to quit attempts and former smoking. J Adolesc Health 2014;54:684-90. 


\section{Articles | wang et al.}

4. Wang MP, Ho SY, Leung LT, Lam TH. Electronic cigarette use and its association with smoking in Hong Kong Chinese adolescents. Addict Behav 2015;50:124-7.

5. Amato MS, Boyle RG, Levy D. How to define e-cigarette prevalence? Finding clues in the use frequency distribution. Tob Control 2016;25:e24-9.

6. Dutra LM, Glantz SA. Electronic cigarettes and conventional cigarette use among US adolescents: a cross-sectional study. JAMA Pediatr 2014;168:610.

7. Malas M, Tempel J van der, Schwartz R, et al. Electronic cigarettes for smoking cessation: a systematic review. Nicotine Tob Res 2016;18: 1926-36.

8. Kalkhoran S, Glantz SA. E-cigarettes and smoking cessation in real-world and clinical settings: a systematic review and meta-analysis. Lancet Respir Med 2016;4:116-28.

9. Ramo DE, Young-Wolff KC, Prochaska JJ. Prevalence and correlates of electronic-cigarette use in young adults: findings from three studies over five years. Addict Behav 2015;41:142-7.

10. Simon P, Kong G, Cavallo DA, Krishnan-Sarin S. Update of adolescent smoking cessation interventions: 2009-2014. Curr Addict Rep 2015;2: $15-23$.

11. Lippert AM. Do adolescent smokers use E-cigarettes to help them quit? The sociodemographic correlates and cessation motivations of U.S. adolescent E-cigarette use. Am J Health Promot 2015;29:374-9.

12. Park J-Y, Seo D-C, Lin H-C. E-cigarette use and intention to initiate or quit smoking among US youths. Am J Public Health 2016;106:672-8.

13. Bullen C, Howe C, Laugesen M, et al. Electronic cigarettes for smoking cessation: a randomised controlled trial. Lancet 2013;382:1629-37.

14. Caponnetto P, Campagna D, Cibella F, et al. EffiCiency and safety of an eLectronic cigAreTte (ECLAT) as tobacco cigarettes substitute: a prospective 12-month randomized control design study. PLoS ONE 2013;8:e66317.

15. Tseng T-Y, Ostroff JS, Campo A, et al. A randomized trial comparing the effect of nicotine versus placebo electronic cigarettes on smoking reduction among young adult smokers. Nicotine Tob Res 2016;18:1937-43.

16. Dawkins L, Kimber C, Puwanesarasa Y, Soar K. First- versus secondgeneration electronic cigarettes: predictors of choice and effects on urge to smoke and withdrawal symptoms. Addiction 2015;110:669-77.

17. Lechner WV, Meier E, Wiener JL, et al. The comparative efficacy of firstversus second-generation electronic cigarettes in reducing symptoms of nicotine withdrawal. Addiction 2015;110:862-7.

18. Hitchman SC, Brose LS, Brown J, Robson D, McNeill A. Associations between e-cigarette type, frequency of use, and quitting smoking: findings from a Longitudinal Online Panel Survey in Great Britain. Nicotine Tob Res 2015;17:1187-94.

19. Stanton A, Grimshaw G. Cochrane Database of Systematic Reviews. John Wiley \& Sons, 2013.

20. Pepper JK, Ribisl KM, Emery SL, Brewer NT. Reasons for starting and stopping electronic cigarette use. Int J Environ Res Public Health 2014;11: 10345-61.

21. McQueen A, Tower S, Sumner W. Interviews with "Vapers": implications for future research with electronic cigarettes. Nicotine Tob Res 2011;13: 860-7.

22. Leventhal AM, Strong DR, Kirkpatrick MG, et al. Association of electronic cigarette use with initiation of combustible tobacco product smoking in early adolescence. JAMA 2015;314:700.

23. Wang MP, Ho SY, Leung LT, Lam TH. Electronic cigarette use and respiratory symptoms in Chinese adolescents in Hong Kong. JAMA Pediatr 2016;170:89.

24. Wong DCN, Chan SSC, Lam T. Depressive symptoms delayed quit attempts and shortened abstinence in young smokers of the Hong Kong youth quitline. Nicotine Tob Res 2016;18:251-8.

25. Li WHC, Chan SSC, Wang MP, et al. An evaluation of the youth quitline service young Hong Kong smokers. J Adolesc Health 2017;60:584-91.

26. SRNT Subcommittee on Biochemical Verification. Biochemical verification of tobacco use and cessation. Nicotine Tob Res 2002;4:149-59.

27. Heatherton TF, Kozlowski LT, Frecker RC, Fagerström KO. The Fagerström test for nicotine dependence: a revision of the Fagerström tolerance questionnaire. Br J Addict 1991;86:1119-27.

28. Lam T-H, Abdullah ASM, Chan SSC, Hedley AJ. Hong Kong Council on Smoking and Health Smoking Cessation Health Centre (SCHC) Steering Group. Adherence to nicotine replacement therapy versus quitting smoking among Chinese smokers: a preliminary investigation. Psychopharmacology 2005;177:400-8.

29. Prochaska JO, Velicer WF. The transtheoretical model of health behavior change. Am J Health Promot 1997;12:38-48.

30. Rollnick S. Readiness, importance, and confidence: critical conditions of change in treatment. In: Miller WR, Heather $\mathrm{N}$, eds. Treating Addictive Behaviors. 2nd edn. New York, NY, USA: Plenum Press, 1998:49-60.

31. Centersfor. Disease Control and Prevention (CDC). Cigarette smoking among adults-United States, 1999. MMWR 2001;50:869-73.

32. Patrick DL, Cheadle A, Thompson DC, Diehr P, Koepsell T, Kinne S. The validity of self-reported smoking: a review and meta-analysis. Am J Public Health 1994;84:1086-93. 